Address correspondence to: Richard L. Proia, Building 10, Room 9N-314, National Institutes of Health, 10 Center Drive MSC 1821, Bethesda, Maryland 208921821, USA. Phone: (301) 496-4391; Fax: (301) 496-9878; E-mail: proia@nih.gov.

\footnotetext{
1. Scriver, C.R., et al., editors. 2001. In The metabolic and molecular bases of inherited disease. McGraw-Hill. New York, New York, USA. 3371-3896.

2. Brady, R.O. 2003. Enzyme replacement therapy: conception, chaos and culmination. Philos. Trans. $R$. Soc. Lond. B Biol. Sci. 358:915-919.

3. Kakkis, E.D., et al. 2001. Enzyme-replacement therapy in mucopolysaccharidosis I. N. Engl. J. Med. 344:182-188.

4. Brooks, A.I., et al. 2002. Functional correction of established central nervous system deficits in an animal model of lysosomal storage disease with feline immunodeficiency virus-based vectors. Proc.
}

Natl. Acad. Sci. U. S. A. 99:6216-6221.

5. Taylor, R.M., and Wolfe, J.H. 1997. Decreased lysosomal storage in the adult MPS VII mouse brain in the vicinity of grafts of retroviral vector-corrected fibroblasts secreting high levels of beta-glucuronidase. Nat. Med. 3:771-774.

6. Snyder, E.Y., Taylor, R.M., and Wolfe, J.H. 1995 Neural progenitor cell engraftment corrects lysosomal storage throughout the MPS VII mouse brain. Nature. 374:367-370.

7. Platt, F.M., et al. 1997. Prevention of lysosomal storage in Tay-Sachs mice treated with N-butyldeoxynojirimycin. Science. 276:428-431.

8. Fan, J.Q., Ishii, S., Asano, N., and Suzuki, Y. 1999. Accelerated transport and maturation of lysosomal alpha-galactosidase A in Fabry lymphoblasts by an enzyme inhibitor. Nat. Med. 5:112-115.

9. Krivit, W., et al. 1998. Hematopoietic stem-cell transplantation in globoid-cell leukodystrophy. N. Engl. J. Med. 338:1119-1126.

10. Biffi, A., et al. 2004. Correction of metachromatic leukodystrophy in the mouse model by trans- plantation of genetically modified hematopoietic stem cells. J. Clin. Invest. 113:1118-1129. doi:10.1172/JCI200419205

11. Eglitis, M.A., and Mezey, E. 1997. Hematopoietic cells differentiate into both microglia and macroglia in the brains of adult mice. Proc. Natl. Acad. Sci. U. S. A. 94:4080-4085.

12. Mezey, E., Chandross, K.J., Harta, G., Maki, R.A., and McKercher, S.R. 2000. Turning blood into brain: cells bearing neuronal antigens generated in vivo from bone marrow. Science. 290:1779-1782.

13. Brazelton, T.R., Rossi, F.M., Keshet, G.I., and Blau, H.M. 2000. From marrow to brain: expression of neuronal phenotypes in adult mice. Science. 290:1775-1779.

14. Priller, J., et al. 2001. Targeting gene-modified hematopoietic cells to the central nervous system: use of green fluorescent protein uncovers microglial engraftment. Nat. Med. 7:1356-1361.

15. Wada, R., Tifft, C.J., and Proia, R.L. 2000. Microglial activation precedes acute neurodegeneration in Sandhoff disease and is suppressed by bone marrow transplantation. Proc. Natl. Acad. Sci. U. S. A. 97:10954-10959.

\title{
Undermining the endothelium by ablation of MAPK-MEF2 signaling
}

Eric N. Olson

Department of Molecular Biology, The University of Texas Southwestern Medical Center at Dallas, Dallas, Texas, USA.

\begin{abstract}
Numerous stimuli activate Big MAPK-1 (BMK1), an MAPK that activates the myocyte enhancer factor-2 (MEF2) transcription factor. Conditional gene deletion showed BMK1 to be required for survival of endothelial cells (see the related article beginning on page 1138). An active form of MEF2C could partially bypass the requirement for BMK1 for endothelial cell survival in vitro. These findings reveal an essential role for BMK1-MEF2 signaling in an endothelial cell survival pathway and raise interesting questions about the molecular basis of this response.
\end{abstract}

A variety of extracellular stimuli transmit signals from the cell membrane to the nucleus via a cascade of MAPKs (1). MAPK pathways contain three distinct types of MAPKs that are activated sequentially (Figure 1). The most upstream kinase, MAPK kinase kinase (MEKK), is activated by $\mathrm{G}$ protein-coupled receptors and phosphorylates a subordinate MAPK kinase (MEK), which phosphorylates an MAPK. The final MAPK in the cascade phosphorylates various transcription factors, leading to activation of specific programs of gene expression.

Nonstandard abbreviations used: big MAPK-1 (BMK1); embryonic day (E); endothelial cell (EC); MAPK kinase (MEK); MAPK kinase kinase (MEKK); myocyte enhancer factor-2 (MEF2); polyinosinic-polycytidylic acid (pIpC).

Conflict of interest: The author has declared that no conflict of interest exists.

Citation for this article:

J. Clin. Invest. 113:1110-1112 (2004).

doi:10.1172/JCI200421497.
Big MAPK-1 (BMK1; also called ERK5) is the terminal MAPK that is activated by MEK5, which is activated by MEKK2/3 (Figure 1). BMK1 is unique among MAPKs because of its large size and bifunctionality. The $\mathrm{N}$-terminal region of BMK1 contains the kinase domain, while the C-terminal region functions as a transcription activation domain (2). One of the best-characterized targets of BMK1 is the myocyte enhancer factor-2 (MEF2) family of transcription factors (3-6). There are four mammalian $M E F 2$ genes, $M E F 2 A,-B,-C$, and $-D$, which are expressed in overlapping patterns in numerous cell types (7). MEF2 factors bind DNA as homo- and heterodimers and activate or repress transcription by recruiting positive or negative cofactors, many of which are cell type-specific and signal-responsive. First discovered as regulators of muscle development, MEF2 factors are now known to play diverse roles in the control of cell growth, survival, and apoptosis (7).

\section{BMK1 acts in an endothelial cell survival pathway}

Previous studies showed that KO mice lacking BMK1 die around embryonic day (E) 10 from severe abnormalities in cardiovascular development that are remarkably similar to defects seen in $\mathrm{KO}$ mice lacking MEKK3 or MEF2C, consistent with the sequential actions of these signaling molecules (8-13). However, the severity of the defects in these mutant embryos, and the associated abnormalities in yolk sac development, complicated the interpretation of the phenotypes and precluded the identification of the precise cell type responsible for embryonic lethality.

To further define the role of BMK1 in mouse development, Hayashi and coworkers generated mice harboring a conditional $B M K 1$ allele (14). In this issue of the JCI, they report that excision of $B M K 1$ after birth, using an inducible Cre recombinase transgene controlled by polyinosinic-polycytidylic (pIpC) acid, resulted in degeneration of the cardiovascular system, accompanied by multifocal hemorrhages, distended capillaries, and ruptures in the normally seamless endothelial lining of the vessels and the 


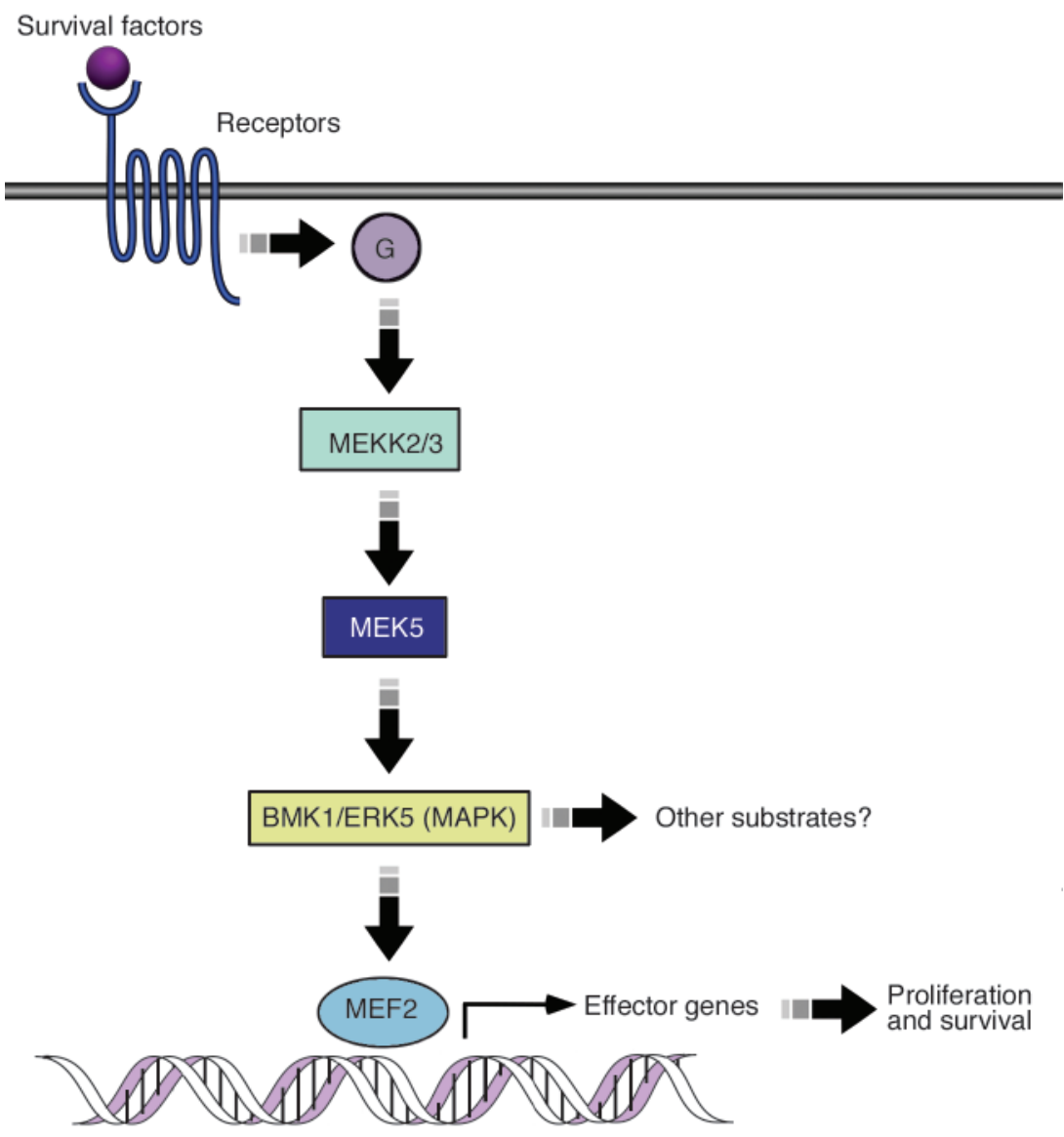

Figure 1

MAPK signaling pathways. Survival signals activate MEKK2/3, which activates MEK5, which activates BMK1. BMK1 stimulates the transcriptional activity of MEF2C by phosphorylating the transcription activation domain and by interacting directly with MEF2C and contributing its own transcriptional activation domain. MEF2C is required for cell survival and proliferation by activating downstream target genes that remain to be identified.

heart. Vascular demise following $B M K 1$ deletion was attributable to apoptosis of endothelial cells (ECs). Subsequent analysis of the expression pattern of the pIpCinducible Cre transgene using a floxed alkaline phosphatase reporter showed strong expression in endothelial and endocardial cells. In agreement with the conclusion that the lethal phenotype reflected an essential role of BMK1 in the endothelium, $B M K 1$ deletion using a Cre transgene controlled by the endothelialspecific Tie 2 promoter caused embryonic lethality at E9.5-10.5 with a phenotype indistinguishable from that of global BMK1-KO mice.

ECs normally proliferate and undergo a mesenchymal transformation in cardiac explant cultures. In contrast, explanted ECs from BMK1-KO mice failed to proliferate in vitro (14). The specific dependence of ECs on BMK1 signaling for proliferation and survival was further demonstrated by the finding that ECs isolated from conditional BMK1-KO mice stopped dividing and underwent apoptosis upon deletion of BMK1 with a Cre-expressing adenovirus in vitro, whereas fibroblasts were unaffected by BMK1 deletion.

VEGF and other growth factors that promote EC survival and proliferation activate BMK1 in cultured ECs. The possibility that MEF2C is a target of BMK1 in an EC survival pathway is supported by the finding that serum, a source of survival signals, stimulated activity of an MEF2C reporter in cultured ECs, and this stimulatory effect was lost upon deletion of BMK1 (14). Moreover, infection of BMK1-KO ECs with an adenovirus encoding a constitutively active form of MEF2C fused to the VP16 activation domain partially protected cells from apoptosis following BMK1 deletion.

\section{A BMK1-MEF2 connection in endothelial survival}

Together, these findings reveal a cellautonomous requirement for BMK1-MEF2 signaling for EC proliferation and viability. The endothelial phenotype of BMK1-KO mice raises interesting questions about the cellular specificity of this response and the targets of BMK1 and MEF2 in the EC survival pathway. For example, it is unclear why ECs are so dependent on the BMK1MEF2C signaling module for survival, whereas fibroblasts and hepatocytes have been shown to be unaffected by deletion of BMK1 (14). Previous studies have shown that BMK1 and MEF2C also promote survival of neurons in vitro (15-18). Perhaps other MAPK and MEF2 family members play redundant roles in other cell types, or perhaps ECs and neurons are more dependent on continual antiapoptotic signals for survival than other cell types. It will be of interest to identify the target genes of $\mathrm{MEF} 2 \mathrm{C}$ in the EC survival pathway and to determine the extent to which BMK1 substrates other than MEF2C contribute to the EC phenotype of BMK1-KO mice. The fact that MEF2C-VP16 protected BMK1-KO ECs only partially from cell death suggests the existence of additional antiapoptotic BMK1 targets.

\section{Other cardiovascular functions of BMK1-MEF2}

The vascular endothelium is a seamless, yet dynamic, tissue required for multiple functions of the cardiovascular system, including maintenance of vascular tone, regulation of blood circulation, coagulation, inflammatory responses, and proper growth and development of vascular smooth muscle and cardiac myocytes. Perturbation of the vascular endothelium is responsible for a variety of cardiovascular disorders, including atherosclerosis, thrombosis, and hypertension. It will be of interest to determine whether BMK1 and MEF2 are involved in the pathogenic mechanisms associated with these endothelial disorders. In this regard, BMK1 is potently activated by fluid shear stress within the vessel wall, which is atheroprotective (19). Conversely, lack of shear stress has been shown to trigger EC apoptosis. Shear stress and activated MEK5 stimulate phosphorylation of the pro-apoptotic factor Bad, which prevents it from translocating to the mitochondria and activating caspase-3. BMK1 is also activated by and plays a protective role against oxidative stress. The apparent requirement 
of MEF2C for maintenance of endothelial integrity is also intriguing in light of the recent association of premature coronary artery disease and myocardial infarction with a mutation in the human $M E F 2 A$ gene (20). Since MEF2A is highly expressed in the endothelium and is a substrate for BMK1, it is likely to act within the same EC survival pathway as MEF2C.

A remarkable number of processes within the cardiovascular system are dependent on signaling from MAPKs to MEF2 (7). In addition to its requirement for EC survival, this signaling pathway is important for differentiation and morphogenesis of cardiac and smooth muscle cells, and has been implicated in numerous cardiovascular disorders, including cardiac hypertrophy, dilated cardiomyopathy, coronary artery disease, and myocardial infarction. Further insights into the functions and mechanisms of action of this signaling module promise to provide new opportunities for its therapeutic manipulation in the settings of human disease.

Address correspondence to: Eric N. Olson, Department of Molecular Biology, The University of Texas Southwestern Medical Center at Dallas, 5323 Harry Hines Blvd., NA8.602, Dallas, Texas 75390-9148, USA. Phone: (214) 648-1187; Fax: (214) 648-1196; E-mail: eric.olson@UTSouthwestern.edu.
1. Raman, M., and Cobb, M.H. 2003. MAP kinase modules: many roads home. Curr. Biol. 13:R886-R888.

2. Kasler, H.G., Victoria, J., Duramad, O., and Winoto, A. 2000. ERK5 is a novel type of mitogen-activated protein kinase containing a transcriptional activation domain. Mol. Cell. Biol. 20:8382-8389.

3. Yang, C.C., Ornatsky, O.I., McDermott, J.C., Cruz, T.F., and Prody, C.A. 1998. Interaction of myocyte enhancer factor 2 (MEF2) with a mitogen-activated protein kinase, ERK5/BMK1. Nucleic Acids Res. 26:4771-4777

4. Marinissen, M.J., Chiariello, M., Pallante, M., and Gutkind, J.S. 1999. A network of mitogen-activated protein kinases links $\mathrm{G}$ protein-coupled receptors to the c-jun promoter: a role for c-Jun NH2-terminal kinase, p38, and extracellular signal-regulated kinase 5. Mol. Cell. Biol. 19:4289-4301.

5. Fukuhara, S., Marinissen, M.J., Chiariello, M., and Gutkind, J.S. 2000. Signaling from G protein-coupled receptors to ERK5/Big MAPK 1 involves Galpha $q$ and Galpha 12/13 families of heterotrimeric $G$ proteins. Evidence for the existence of a novel Ras and Rho-independent pathway. J. Biol. Chem. 275:21730-21736.

6. Kato, Y., et al. 2000. Big mitogen-activated protein kinase regulates multiple members of the MEF2 protein family. J. Biol. Chem. 275:18534-18540.

7. McKinsey, T.A., Zhang, C.L., and Olson, E.N. 2002 MEF2: a calcium-dependent regulator of cell division, differentiation and death. Trends Biochem. Sci. 27:40-47.

8. Regan, C.P., et al. 2002. Erk5 null mice display multiple extraembryonic vascular and embryonic cardiovascular defects. Proc. Natl. Acad. Sci. U. S. A. 99:9248-9253.

9. Sohn, S.J., Sarvis, B.K., Cado, D., and Winoto, A 2002. ERK5 MAPK regulates embryonic angiogenesis and acts as a hypoxia-sensitive repressor of vascular endothelial growth factor expression. J. Biol. Chem. 277:43344-43351.

10. Yang, J., et al. 2000. Mekk3 is essential for early embryonic cardiovascular development. Nat. Genet. 24:309-313.
11. Lin, Q., Schwarz, J., Bucana, C., and Olson, E.N. 1997. Control of mouse cardiac morphogenesis and myogenesis by transcription factor MEF2C. Science. 276:1404-1407.

12. Lin, Q., et al. 1998. Requirement of the MADS-box transcription factor MEF2C for vascular development. Development. 125:4565-4574.

13. Bi, W., Drake, C.J., and Schwarz, J.J. 1999. The transcription factor MEF2C-null mouse exhibits complex vascular malformations and reduced cardiac expression of angiopoietin 1 and VEGF. Dev. Biol. 211:255-267.

14. Hayashi, M., et al. 2004. Targeted deletion of $B M K 1 / E R K 5$ in adult mice perturbs vascular integrity and leads to endothelial failure. J. Clin. Invest. 113:1138-1148. doi:10.1172/JCI200419890.

15. Liu, L., et al. 2003. ERK5 activation of MEF2-mediated gene expression plays a critical role in BDNFpromoted survival of developing but not mature cortical neurons. Proc. Natl. Acad. Sci. U. S. A. 100:8532-8537

16. Linseman, D.A., et al. 2003. Inactivation of the myocyte enhancer factor-2 repressor histone deacetylase- 5 by endogenous $\mathrm{Ca}^{2+} / /$ calmodulindependent kinase II promotes depolarization-mediated cerebellar granule neuron survival. J. Biol. Chem. 278:41472-41481.

17. Mao, Z., Bonni, A., Xia, F., Nadal-Vicens, M., and Greenberg, M.E. 1999. Neuronal activity-dependent cell survival mediated by transcription factor MEF2. Science. 286:785-790.

18. Li, M., et al. 2001. Myocyte enhancer factor $2 \mathrm{~A}$ and $2 \mathrm{D}$ undergo phosphorylation and caspase-mediated degradation during apoptosis of rat cerebellar granule neurons. J. Neurosci. 21:6544-6552.

19. Pi, X., Yan, C., and Berk, B.C. 2004. Big mitogenactivated protein kinase (BMK1)/ERK5 protects endothelial cells from apoptosis. Circ. Res. 94:362-369.

20. Wang, L., Fan, C., Topol, S.E., Topol, E.J., and Wang, Q. 2003. Mutation of MEF2A in an inherited disorder with features of coronary artery disease. Science. 302:1578-1581.

\title{
Insig: a significant integrator of nutrient and hormonal signals
}

\author{
Alan D. Attie \\ Department of Biochemistry, University of Wisconsin-Madison, Madison, Wisconsin, USA.
}

\begin{abstract}
Lipogenesis is regulated by sterols and by insulin through the regulated expression and activation of the sterol regulatory element-binding proteins (SREBPs). A new study shows one way in which sterol and insulin regulation can be decoupled (see the related article beginning on page 1168). In transgenic mice overexpressing a protein that regulates SREBP activation, lipogenesis is more sensitive to cholesterol and less sensitive to insulin.
\end{abstract}

\author{
Nonstandard abbreviations used: insulin receptor \\ substrate-2 (IRS-2); SREBP cleavage-activating protein \\ (SCAP); sterol regulatory element-binding \\ protein/adipocyte differentiation factor-1 \\ (SREBP/ADD1). \\ Conflict of interest: The author has declared that no \\ conflict of interest exists. \\ Citation for this article: \\ J. Clin. Invest. 113:1112-1114 (2004). \\ doi:10.1172/JCI200421450.
}

Animal cells, like nations, have a domestic and a foreign policy. "Domestic policy" involves local control of metabolism, responses to energy charge, redox status, or the concentration of key allosteric regulators (e.g., malonyl-CoA and fructose 2,6-bisphosphate). The world of a multicellular organism imposes a "foreign policy" on its cells. They must respond to global signals in the interest of the whole organism.

Global and local signals invariably converge (as former Congressman Tip O'Neil famously quipped, "All politics is local."). Lipogenesis is a case in point. Feeding, fasting, and refeeding cause large changes in the rates of fatty acid and cholesterol synthesis. These changes are evoked by both the carbohydrate and the lipid components of the diet. We now have a molecular window into some of the key molecules that integrate nutrient-derived signals.

A key breakthrough was the simultaneous discovery by Tontonoz et al. (1) and Yokoyama et al. (2) of the sterol regulatory ele- 